\section{Comportamiento antibacteriano de partículas de cobre frente a microorganismos obtenidos de úlceras crónicas infectadas y su relación con la resistencia a antimicrobianos de uso común}

\author{
NALDY FEBRÉ ${ }^{1, \mathrm{a}}$, VIVIANA SILVA ${ }^{2, \mathrm{~b}}$, ANDREA BÁEZ $^{3, \mathrm{c}}$, \\ HUMBERTO PALZA ${ }^{4, d}$, KATHERINE DELGADO ${ }^{4, e}$, \\ ISABEL ABURTO ${ }^{5, \mathrm{f}}$, VICTOR SILVA ${ }^{6, \mathrm{~g}}$
}

\section{Antibacterial activity of copper salts against microorganisms isolated from chronic infected wounds}

Background: The antimicrobial activity of copper $\left(\mathrm{Cu}^{+2}\right)$ is recognized and used as an antimicrobial agent. Aim: To evaluate the antimicrobial activity of copper against microorganisms obtained from chronic cutaneous wound infections. Material and Methods: Five chemical products that contained copper particles in their composition were tested (zeolite, silica, acetate, nitrate and nanoparticle of copper). The antimicrobial activity against antibiotic resistant strains usually isolated from chronic cutaneous wound infections was determined for two of the products with better performance in copper release. Results: The minimal inhibitory and minimal bactericidal concentrations of copper acetate and nitrate were similar, fluctuating between $400-2,000 \mu \mathrm{g} / \mathrm{ml}$. Conclusions: The studied copper salts show great potential to be used to control both gram positive and gram negative, antibiotic resistant bacteria isolated from wound infections.

(Rev Med Chile 2016; 144: 1523-1530)

Key words: Anti-Bacterial Agents; Anti-Infective Agents; Copper; Drug Resistance, Microbial; Skin Ulcer.

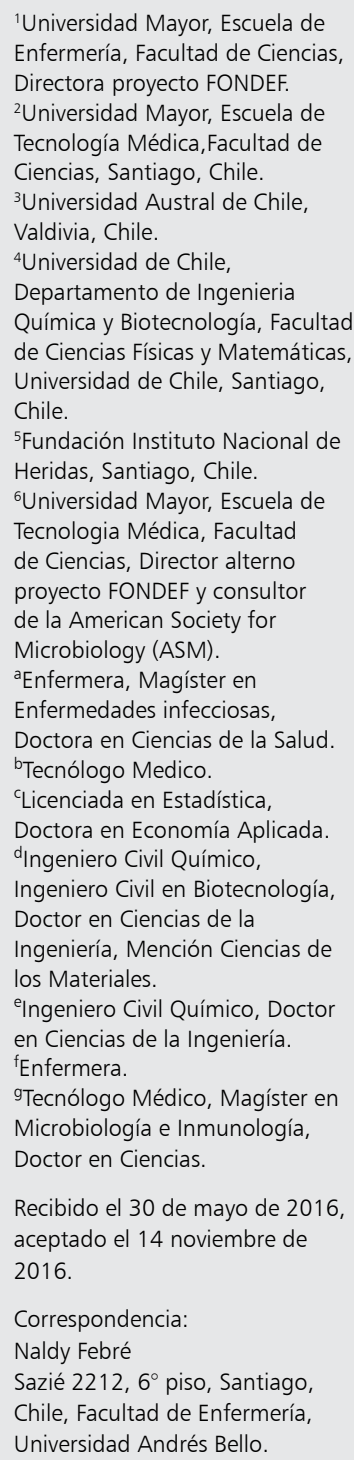

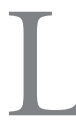
as úlceras cutáneas crónicas constituyen un problema sanitario relevante a nivel mundial, se calcula que entre $1 \%$ al $2 \%$ de la población padecerá sus consecuencias durante su vida ${ }^{1}$. En Latinoamérica, alrededor del $1 \%$ de la población general presenta úlceras abiertas y cicatrizadas; $25 \%$ de ellas corresponden a úlceras venosas activas ${ }^{2}$. En Chile, las estadísticas disponibles muestran que aproximadamente 160.000 pacientes son portadores de algún tipo de herida o úlcera, constituyéndose en un problema de salud pública ${ }^{3}$ Se define úlcera crónica a "una lesión de piel mantenida en el tiempo, que implica pérdida de la epidermis, parte de la dermis e incluso hipodermis", encontrándose en este grupo a las úlceras venosas, úlceras isquémicas, pie diabético, úlceras por presión, quemaduras y heridas quirúrgicas infectadas ${ }^{4}$.

Todas estas injurias repercuten en la calidad de vida de las personas afectadas, tanto en el ámbito familiar y psicológico, como en el laboral y económico. La atención profesional no opor- 
tuna, produce períodos de incapacidad laboral prolongados, tratamientos costosos, hospitalizaciones reiteradas y cirugías que pueden llegar a la amputación; la ulceración del pie afecta entre un $15 \%$ a $20 \%$ de todos los individuos con diabetes y precede hasta un $85 \%$ de las amputaciones en este grupo ${ }^{9-12}$ la tasa de mortalidad asociada a una infección generalizada en un paciente diabético alcanza un $5 \%{ }^{5-8}$.

Diversos estudios han demostrado que las cepas que afectan a los pacientes portadores de pie diabético incluyen Staphylococcus aureus, Staphylococcus epidermidis, Streptococcus spp, Enterococcus spp, Pseudomonas aeruginosa, y bacterias coliformes, así como Peptostreptococcus, sugiriendo que infecciones polimicrobianas pueden repercutir en aspectos como la virulencia y llevar a que estos microorganismos en comunidad dificulten la recuperación y cicatrización de la lesión ${ }^{13,14}$.

La actividad antimicrobiana de iones de cobre, específicamente de $\mathrm{Cu}^{+2}$ es reconocida en la literatura desde el año 1993, y su actividad sobre los microorganismos es función de su concentración, actuando como un agente bacteriostático en concentraciones entre 25 a $150 \mu \mathrm{M}$ o bactericida en concentraciones superiores a esta. $\mathrm{El} \mathrm{Cu}^{+2}$ interviene directamente: 1) inhibiendo la síntesis de proteínas; 2) alterando la membrana celular del microorganismo causando peroxidación, ya que induce daño oxidativo de lípidos que son claves en el intercambio de moléculas del medio intra y extracelular; 3 ) alterando o destruyendo los ácidos nucleicos de bacterias y virus ${ }^{15-17}$.

Nanopartículas de cobre metálico (NPCu) y nanopartículas de óxidos de cobre (NPCuO) son activas contra diferentes agentes patógenos de infecciones asociadas a la atención de salud $(\text { IAAS })^{18}$. Con el propósito de crear materiales multifuncionales, diversas investigaciones han comprobado que incorporar nanopartículas de cobre en matrices orgánicas inertes frente a la contaminación bacteriana, produce un material compuesto antimicrobiano ${ }^{17-24}$. Estudios que evaluaron la efectividad del cobre sobre S. epidermidis, recuperados de implantes de silicona utilizados para el aumento mamario, concluyen que los implantes con aditivos de cobre impiden la adhesión del S. epidermidis ${ }^{25-28}$.

Es por lo anterior, que la presente investigación tiene como objetivo determinar el comportamiento antibacteriano de partículas de cobre frente a microorganismos obtenidos de úlceras crónicas infectadas y para así determinar su relación con la resistencia a antimicrobianos de uso común.

\section{Materiales y Métodos}

Se planteó un estudio prospectivo iniciándose con la creación de un banco de cepas aisladas de muestras clínicas de pacientes portadores de heridas cutáneas crónicas infectadas (se consideró infección cuando existió un recuento mayor o igual a 100.000 bacterias por gramo de tejido obtenido de úlcera). El tamaño muestral se obtuvo en base a la prevalencia de heridas y úlceras en la población adulta en Chile ( 8 millones de personas), equivale a $2 \%$, Nivel de confianza de $99 \%$, estimándose un error de muestreo del $3 \%$ y se utilizó como fuente de datos la encuesta epidemiológica del Ministerio de Salud, año $2000^{29}$. Los pacientes fueron invitados a participar en la Fundación Instituto Nacional de Heridas (INH). Los criterios de inclusión del estudio fueron los siguientes: paciente mayor a 18 años, portador de una úlcera cutánea crónica infectada (úlcera venosa, úlcera hipertensiva o pie diabético), cuya infección no fuera resistente a antimicrobianos, no haber estado hospitalizado en los últimos seis meses, y aceptar mediante una firma el consentimiento informado.

Cada muestra clínica representativa de la infección fue obtenida por un profesional de enfermería entrenado, previo lavado por arrastre con suero fisiológico estéril o agua bidestilada, se frotó con tórula estéril el centro y los bordes internos en zigzag en aproximadamente 10 puntos de referencia de la superficie comprometida, depositando la tórula en medio de transporte Stuart ${ }^{4}$, siendo transportada al Laboratorio de Investigación Microbiológica de la Universidad Mayor, en un plazo inferior a dos horas. Cada tórula fue sembrada en placas de Petri con agar Sangre de cordero, agar chocolate y Agar MacConkey, las cuales fueron incubadas a $35-37^{\circ} \mathrm{C}$ por $24 \mathrm{~h}$. Paralelamente, se realizó un frotis en portaobjeto sometido a tinción de Gram para su observación y análisis microscópico. Luego del periodo de incubación, las placas se analizaron morfológicamente (macro y microscópicamente) para evidenciar cultivo mono o polimicrobiano. Las especies bacterianas se identificaron por pruebas fisiológicas, enzimáticas y/o bioquímicas, empleando galerías comerciales API 
$\left(\right.$ Biomerieux $\left.{ }^{\mathrm{R}}\right)$. La sensibilidad antimicrobiana fue evaluada mediante el método de difusión en agar o Kirby Bauer en medio Muller Hinton, evaluando los siguientes antimicrobianos, para $S$ aureus (cefoxitina, cotrimoxazol, ampicilina-sulbactam, tetraciclina), Enterobacterias (cefalotina, cefuroximo, ceftazidima, gentamicina, amikacina) y para bacilos no fermentadores (BNF) (ceftazidinma, imipenem, amikacina, gentamicina, ampicilina/ sulbactam).

En el Laboratorio de Polímeros de la Universidad de Chile, se realizó un estudio de caracterización morfológico y de liberación de iones $\mathrm{Cu}^{+2}$ de Acetato de cobre monohidratado, Nitrato de cobre trihidratado, Zeolita funcionalizada, Silica Funcionalizada y Nanoparticula de cobre.

Para ello, se testeó la estabilidad en agua de nanopartículas de cobre metálico, nanopartículas de óxidos de cobre $\left(\mathrm{Cu}^{2} \mathrm{O}\right.$ o $\left.\mathrm{CuO}\right)$, sales de cobre y un material nano/micrométrico inerte que soporta nanoestructuras en base a cobre. Se cuantificó la concentración de $\mathrm{Cu}^{+2}$ a través de ensayos de espectrofotometría UV, de manera de determinar la cantidad de partículas o compuestos de cobre necesarias para obtener las concentraciones deseadas.

Se caracterizaron las diferentes partículas de cobre evaluadas mediante difracción de rayos $\mathrm{X}$, microscopía electrónica de barrido (SEM), microscopía electrónica de transmisión (TEM), y espectrometría de masas (ICP-MS). Una vez caracterizadas, fueron seleccionadas aquellas que presentaron el mejor comportamiento (mayor nivel de liberación de cobre en un periodo de $48 \mathrm{~h}$ ), para ser empleadas en los ensayos in vitro de concentración inhibitoria mínima (CIM) y concentración bactericida mínima (CBM) por método de macrodilución. Para esto, los productos en polvo fueron diluidos en caldo Muller Hinton hasta alcanzar las concentraciones finales de uso, de $200 \mu \mathrm{M} / \mathrm{ml}$ a $2.000 \mu \mathrm{M} / \mathrm{ml}$ en tubos tapa rosca, conteniendo 9,9 ml de solución. El inóculo de cada cepa fue generado a partir de cultivos frescos en agar sangre. Colonias fueron diluidas en suero fisiológico estéril hasta alcanzar una densidad óptica (DO) de 0,2 en espectrofotómetro a 600 $\mathrm{nm}$, inoculando 50 ul a cada tubo. La incubación fue a $37^{\circ} \mathrm{C}$ por $24 \mathrm{~h}$ con agitación constante a 200 rpm. La lectura de CIM fue determinada como el tubo con la menor concentración del producto que no evidenció crecimiento (tubo translúcido o sin turbidez) a ojo de los observadores. La CBM se determinó sembrando desde los tubos que no evidenciaban crecimiento, 100 ul en agar Muller Hinton, incubándose a $37^{\circ} \mathrm{C}$ por $24 \mathrm{~h}$, determinándose como CBM la placa obtenida de la menor concentración del producto en la que no se presentó crecimiento bacteriano.

Se emplearon cepas ATCC como control de calidad para los test de identificación bacteriana y ensayos de susceptibilidad antimicrobiana por difusión en agar y por dilución en caldo ( $P$. aeruginosa ATCC 27853 y S. aureus ATCC 25923), siguiendo las recomendaciones del Clinical Laboratory Standard Institute CLSI ${ }^{30}$.

Se cumplieron las normas éticas internacionalmente exigidas, y el presente proyecto fue aprobado por el comité de ética de la Universidad Mayor y comité de Bioseguridad.

\section{Resultados}

Durante el periodo inicial del estudio comprendido entre marzo a junio del 2014, fueron evaluados un total de 71 pacientes que presentaron los criterios de infección de úlcera cutánea crónica (IUCC). La distribución numérica y porcentual según tipo de lesión y de cepas recuperadas se observa en la Tabla 1, donde destaca que el 71,6\% (111/155 cepas) correspondieron a los pacientes portadores de úlceras venosas infectadas. Cabe destacar que la distribución de los cultivos recuperados el $54,2 \%$ de los muestras analizadas correspondieron a infecciones polimicrobianas.

Mediante espectrofotometría UV observamos que Acetato de cobre y Nitrato de cobre presentan liberación temprana y sostenida de $\mathrm{Cu}^{+2}$, hasta las 48 h de evaluación (Tabla 2).

Se determinó el perfil de susceptibilidad de las cepas aisladas por método de difusión en agar,

Tabla 1. Distribución numérica y porcentual según tipo de lesión y número cepas recuperadas de úlceras crónicas infectadas ( $n=155$ cepas)

\begin{tabular}{|lcc|}
\hline Tipo de Lesión & n cepas & \% \\
\hline Úlcera hipertensiva & 6 & 3,9 \\
\hline Úlcera pie diabético & 38 & 24,5 \\
\hline Úlcera venosa & 111 & 71,6 \\
\hline Total & 155 & 100,0 \\
\hline
\end{tabular}


Tabla 2. Liberación de $\mathrm{Cu}^{+2}$ en períodos de cero a 48 h de las cinco partículas de cobre utilizando espectrofotometría UV

\begin{tabular}{|ccccccc|}
\hline $\begin{array}{c}\text { Tiempo } \\
\text { [h] }\end{array}$ & $\begin{array}{c}\text { Zeolita } \\
\text { funcionalizada } \\
\text { [mg/L] }\end{array}$ & $\begin{array}{c}\text { Sílica } \\
\text { funcionalizada } \\
\text { [mg/L] }\end{array}$ & $\begin{array}{c}\text { Nano Cu } \\
\text { [mg/L] }\end{array}$ & $\begin{array}{c}\text { Acetato de } \\
\mathbf{C u} \\
\text { [mg/L] }\end{array}$ & $\begin{array}{c}\text { Nitrato de } \\
\mathbf{C u} \\
\text { [mg/L] }\end{array}$ & Óxido de Cu \\
\hline 0 & 00,00 & 00,00 & 0,00 & 000,00 & 000,00 & 0,00 \\
\hline 4 & 55,15 & 33,86 & 0,15 & 748,72 & 643,53 & 0,11 \\
\hline 24 & 24,06 & 24,49 & 0,89 & 540,46 & 505,96 & 0,73 \\
\hline 48 & 13,84 & 29,39 & 1,38 & 697,61 & 607,75 & 0,66 \\
\hline
\end{tabular}

Tabla 3. Perfil de Sensibilidad de las cepas de $S$. aureus aisladas de úlceras crónicas infectadas seleccionadas

\begin{tabular}{|c|c|c|c|c|c|c|}
\hline Muestra & $\begin{array}{l}\text { Identificación } \\
\text { por especie }\end{array}$ & Ciprofloxacino & Cefoxitina & Cotrimoxazol & $\begin{array}{l}\text { Ampicilina- } \\
\text { Sulbactam }\end{array}$ & Tetraciclina \\
\hline M10 & S. aureus & $R$ & $\mathrm{R}$ & s & S & s \\
\hline M39 & S. aureus & $\mathrm{R}$ & $\mathrm{R}$ & s & s & s \\
\hline M54 & S. aureus & $R$ & S & S & S & $\mathrm{s}$ \\
\hline M27 & S. aureus & s & S & S & S & S \\
\hline ATCC 25923 & S. aureus & S & s & s & s & S \\
\hline
\end{tabular}

S: Sensible R: Resistente.

a seguir se seleccionaron cepas resistentes para evaluar el comportamiento antimicrobiano de las partículas de cobre seleccionadas (Nitrato de cobre tri-hidratado y Acetato de cobre monohidratado), resultados observados en las tablas 3 a 6 .

De las cuatro cepas de $S$. aureus aisladas de úlceras infectadas, destaca que 3 de las 4 cepas presentaron resistencia a quinolonas y betalactámicos (Tabla 3). De acuerdo a las indicaciones del CLSI se propuso la utilización de cefoxitina para determinar multi-resistencia del S. aureus, definiéndose a estas cepas como resistentes a meticilina de la comunidad (SAMR-C).

El perfil de resistencia de las cinco cepas de enterobacterias aisladas desde úlceras crónicas infectadas, destaca una sensibilidad para Amikacina en 4 de 5 cepas, seguido de ceftazidima con una sensibilidad en 2 cepas analizadas. Frente a cefalotina, las enterobacterias presentaron 4 cepas resistentes y uno con resistencia intermedia. Las bacterias Gram negativas son consideradas multiresistentes si son resistentes a tres o más familias de antimicrobianos, de acuerdo a esta premisa en la
Tabla 4, observamos que 4 de las 5 cepas analizadas son multiresistentes.

La susceptibilidad in vitro de cepas de bacilos no fermentadores (BNF) aislados desde úlceras crónicas infectadas más la cepa ATCC $(\mathrm{n}=5$ cepas) se presenta en la Tabla 5, donde destaca que la resistencia a las quinolonas (ciprofloxacino) y betalactámicos (ceftazidima) está presente en las 4 cepas clínicas, siendo consideradas además multiresistentes.

La Tabla 6 muestra los resultados de la CIM y CBM de las dos partículas de cobre seleccionadas. La CIM para Acetato de cobre tiene un promedio de $826,6 \mu \mathrm{g} / \mathrm{ml}$, con rangos de 400 a $1.200 \mu \mathrm{g} / \mathrm{ml}$ y moda de $1.000 \mu \mathrm{g} / \mathrm{ml}$. Para el nitrato de cobre tri-hidratado la CIM promedio es de 1.133,3 $\mu \mathrm{g} /$ $\mathrm{ml}$, con rangos de 400 a $1.800 \mu \mathrm{g} / \mathrm{ml}$ y moda de $1.000 \mu \mathrm{g} / \mathrm{ml}$, para la cepa de $S$. aureus M10 considerada resistente, la CIM y CBM de cobre fluctuó entre 400 y $600 \mu \mathrm{g} / \mathrm{ml}$ respectivamente, por su parte la cepa E. coli M19C1 sensible solo a amikacina presentó CIM y CBM entre $800 \mu \mathrm{g} / \mathrm{ml}$ a $1.400 \mu \mathrm{g} / \mathrm{ml}$. 
Comportamiento antibacteriano de partículas de cobre - N. Febré et al

Tabla 4. Perfil de Sensibilidad de las cepas de Enterobacterias aisladas de úlceras crónicas infectadas seleccionadas

\begin{tabular}{|c|c|c|c|c|c|c|c|}
\hline Muestra & $\begin{array}{l}\text { Identificación } \\
\text { por especie }\end{array}$ & Ciprofloxacino & Cefalotina & Cefuroximo & Ceftazidima & Gentamicina & Amikacina \\
\hline M19-C1 & E. coli & $\mathrm{R}$ & $\mathrm{R}$ & $\mathrm{R}$ & $\mathrm{R}$ & $\mathrm{R}$ & $\mathrm{s}$ \\
\hline $\mathrm{M} 21-\mathrm{C} 1$ & E. coli & $S$ & I & 1 & $S$ & $\mathrm{R}$ & S \\
\hline M64-C3 & E. coli & $R$ & $\mathrm{R}$ & $\mathrm{R}$ & $\mathrm{R}$ & $S$ & $\mathrm{~s}$ \\
\hline M17-C2 & Providencia rettgeri & R & $\mathrm{R}$ & $\mathrm{R}$ & $\mathrm{R}$ & $\mathrm{R}$ & $\mathrm{s}$ \\
\hline M45-C1 & Proteus mirabilis & $\mathrm{R}$ & $\mathrm{R}$ & S & s & $\mathrm{R}$ & $\mathrm{R}$ \\
\hline
\end{tabular}

S: Sensible I: Intermedio R: Resistente.

Tabla 5. Perfil de Sensibilidad de las cepas de Bacilos no Fermentadores aisladas de úlceras crónicas infectadas seleccionadas

\begin{tabular}{|clcccccc|}
\hline Muestra & $\begin{array}{l}\text { Identificación por } \\
\text { especie }\end{array}$ & $\begin{array}{c}\text { Cipro- } \\
\text { floxacino }\end{array}$ & $\begin{array}{c}\text { Ceftazi- } \\
\text { dima }\end{array}$ & $\begin{array}{c}\text { Imipe- } \\
\text { nem }\end{array}$ & $\begin{array}{c}\text { Amika- } \\
\text { cina }\end{array}$ & $\begin{array}{c}\text { Gentami- } \\
\text { cina }\end{array}$ & $\begin{array}{c}\text { Ampicilina } \\
\text { Sulbactam }\end{array}$ \\
\hline M8 & Pseudomonas aeruginosa & $R$ & $R$ & $R$ & $S$ & $S$ & $/$ \\
M59 & Pseudomonas aeruginosa & $R$ & $R$ & $R$ & $S$ & $S$ & $/$ \\
\hline M65-C3 & Pseudomonas aeruginosa & $R$ & $R$ & $S$ & $R$ & $R$ & $/$ \\
M16 & Acinetobacter baumannii & $R$ & $R$ & $S$ & I & $R$ & $S$ \\
\hline ATCC 27853 & Pseudomonas aeruginosa & $S$ & $S$ & $S$ & $S$ & $S$ & $/$ \\
\hline
\end{tabular}

S: Sensible I: Intermedio R: Resistente.

Tabla 6. Concentración Inhibitoria Mínima (CIM) y Concentración Bactericida Mínima (CBM) de Nitrato de cobre tri-hidratado y Acetato de cobre monohidratado sobre cepas bacterianas ATCC y aisladas de pacientes con úlceras crónicas infectadas

\begin{tabular}{|c|c|c|c|c|}
\hline \multirow[t]{2}{*}{ Cepa } & \multicolumn{2}{|c|}{ CIM } & \multicolumn{2}{|c|}{ CBM } \\
\hline & $\begin{array}{l}\text { Nitrato de cobre } \\
\qquad 3 \mathrm{H}_{2} \mathrm{O}\end{array}$ & $\begin{array}{l}\text { Acetato de cobre } \\
\qquad \mathrm{H}_{2} \mathrm{O}\end{array}$ & $\begin{array}{l}\text { Nitrato de cobre } \\
\qquad 3 \mathrm{H}_{2} \mathrm{O}\end{array}$ & $\begin{array}{l}\text { Acetato de cobre } \\
\qquad \mathrm{H}_{2} \mathrm{O}\end{array}$ \\
\hline S. aureus M10 & $400 \mu \mathrm{g} / \mathrm{ml}$ & $400 \mu \mathrm{g} / \mathrm{ml}$ & $600 \mu \mathrm{g} / \mathrm{ml}$ & $400 \mu \mathrm{g} / \mathrm{ml}$ \\
\hline S. aureus $\mathrm{M} 27$ & $1.400 \mu \mathrm{g} / \mathrm{ml}$ & $1.000 \mu \mathrm{g} / \mathrm{ml}$ & $1.600 \mu \mathrm{g} / \mathrm{ml}$ & $1.600 \mu \mathrm{g} / \mathrm{ml}$ \\
\hline S. aureus M-39 & $1.000 \mu \mathrm{g} / \mathrm{ml}$ & $600 \mu \mathrm{g} / \mathrm{ml}$ & $1.000 \mu \mathrm{g} / \mathrm{ml}$ & $1.000 \mu \mathrm{g} / \mathrm{ml}$ \\
\hline S. aureus M-54 & $1.000 \mu \mathrm{g} / \mathrm{ml}$ & $1.200 \mu \mathrm{g} / \mathrm{ml}$ & $1.400 \mu \mathrm{g} / \mathrm{ml}$ & $1.600 \mu \mathrm{g} / \mathrm{ml}$ \\
\hline E. coli M19-C1 & $1.000 \mu \mathrm{g} / \mathrm{ml}$ & $800 \mu \mathrm{g} / \mathrm{ml}$ & $1.200 \mu \mathrm{g} / \mathrm{ml}$ & $1.400 \mu \mathrm{g} / \mathrm{ml}$ \\
\hline E. coli M21-C1 & $1.400 \mu \mathrm{g} / \mathrm{ml}$ & $1.000 \mu \mathrm{g} / \mathrm{ml}$ & $1.800 \mu \mathrm{g} / \mathrm{ml}$ & $1.600 \mu \mathrm{g} / \mathrm{ml}$ \\
\hline E. coli M64-C3 & $1.200 \mu \mathrm{g} / \mathrm{ml}$ & $600 \mu \mathrm{g} / \mathrm{ml}$ & $2.000 \mu \mathrm{g} / \mathrm{ml}$ & $1.000 \mu \mathrm{g} / \mathrm{ml}$ \\
\hline P. rettgeri $\mathrm{M} 17-\mathrm{C} 2$ & $1.400 \mu \mathrm{g} / \mathrm{ml}$ & $1.000 \mu \mathrm{g} / \mathrm{ml}$ & $1.800 \mu \mathrm{g} / \mathrm{ml}$ & $1.600 \mu \mathrm{g} / \mathrm{ml}$ \\
\hline P. mirabilis M45-C1 & $1.200 \mu \mathrm{g} / \mathrm{ml}$ & $1.000 \mu \mathrm{g} / \mathrm{ml}$ & $1.600 \mu \mathrm{g} / \mathrm{ml}$ & $1.600 \mu \mathrm{g} / \mathrm{ml}$ \\
\hline P. aeruginosa M8 & $1.800 \mu \mathrm{g} / \mathrm{ml}$ & $1.000 \mu \mathrm{g} / \mathrm{ml}$ & $2.000 \mu \mathrm{g} / \mathrm{ml}$ & $1.400 \mu \mathrm{g} / \mathrm{ml}$ \\
\hline P. aeruginosa M59 & $1.200 \mu \mathrm{g} / \mathrm{ml}$ & $1.000 \mu \mathrm{g} / \mathrm{ml}$ & $1.600 \mu \mathrm{g} / \mathrm{ml}$ & $1.200 \mu \mathrm{g} / \mathrm{ml}$ \\
\hline$P$. aeruginosa M65-C3 & $1.000 \mu \mathrm{g} / \mathrm{ml}$ & $600 \mu \mathrm{g} / \mathrm{ml}$ & $1.600 \mu \mathrm{g} / \mathrm{ml}$ & $1.000 \mu \mathrm{g} / \mathrm{ml}$ \\
\hline A. baumannii M16 & $1.000 \mu \mathrm{g} / \mathrm{ml}$ & $600 \mu \mathrm{g} / \mathrm{ml}$ & $1.400 \mu \mathrm{g} / \mathrm{ml}$ & $1.000 \mu \mathrm{g} / \mathrm{ml}$ \\
\hline
\end{tabular}




\section{Discusión}

Las úlceras cutáneas crónicas ofrecen un nicho ecológico ideal para la multiplicación microbiana, por la pérdida de integridad cutánea, hipoxia tisular y por contaminación de la lesión expuesta. Siendo la infección de estas, uno de los factores que retrasa su cicatrización, llegando a complicaciones que pueden ser osteomielitis, bacteriemia o celulitis ${ }^{31,32}$.

De acuerdo a los datos obtenidos en la presente investigación, a partir de 155 cepas recuperadas de pacientes que presentaron los criterios de IUCC, destaca que el 71,6\% (111 cepas) correspondieron a los pacientes portadores de úlceras venosas, concordando con lo descrito en la literatura, la que considera a ésta lesión la más frecuente en extremidades, afectando al $1 \%$ de la población general y al 3\% de los mayores de 80 años ${ }^{32,33}$.

Algunos microorganismos presentes en las úlceras crónicas son capaces de formar matriz de polisacáridos y unirse en forma de biopelícula (biofilms) protegiéndolos de los antimicrobianos transformándolos en resistentes debido a su organización in vivo ${ }^{34}$. La infección suele ser de tipo polimicrobiana, siendo $S$. aureus el agente que con más frecuencia se aísla ${ }^{31,35,36}$. El 75\% de las cepas de $S$. aureus recuperados en la presente investigación fueron resistentes a quinolonas, betalactámicos, cefoxitina, evidenciando alta frecuencia de cepas SAMR (Tabla 3). Para las enterobacterias recuperadas la resistencia a amikacina alcanzó un 20\%; destacamos que todas las cepas analizadas no presentaron sensibilidad a cefalotina.

En bacilos no fermentadores (BNF), la susceptibilidad in vitro de cepas clínicas aisladas desde úlceras crónicas infectadas muestran resistencia a las quinolonas (ciprofloxacino) y betalactámicos (ceftazidima), además observamos que las cuatro cepas de $P$. aeruginosa son multirresistentes.

De acuerdo a la literatura, el cobre $(\mathrm{Cu})$ como un metal de transición se produce en la naturaleza en varios estados de oxidación, comúnmente utilizado en forma de sulfato, el sulfato de cobre (I) o sulfato cuproso, es una sal insoluble y poco estable por lo que es menos frecuente que el sulfato de cobre (II), una sal soluble en agua que puede presentarse como sulfato cúprico $\left(\mathrm{CuSo}_{4}\right)$ o sulfato cúprico pentahidratado $\left(\mathrm{CuSO}_{4} 5 \mathrm{H}_{2} \mathrm{O}\right)$, este último es, en la práctica, el de preferencia para ser utilizado como antibacteriano en diversos ámbitos ${ }^{37}$.
La caracterización realizada de las cinco partículas de cobre, permitió determinar que cuatro de ellas presentan un contenido de cobre similar (26 a 36\%), solo dos (acetato de cobre monohidratado y nitrato de cobre Tri-hidratado) presentan liberación inmediata, elevada y prolongada, hasta por lo menos 48 h que duró la medición (Tabla 2). La CIM promedio para acetato de cobre fue igual a $826,6 \mu \mathrm{g} / \mathrm{ml}$ y para el nitrato de cobre trihidratado la CIM promedio fue de $1.133,33 \mu \mathrm{g} / \mathrm{ml}$. Se destaca que, esta cantidad en aplicación tópica en el ser humano es cercana a la recomendación de ingesta diaria establecida por la Junta de Nutrición y Alimentos del Instituto de Medicina, cifra que alcanza a los 900 microgramos/día.

En conclusión, la presente investigación establece la caracterización morfológica de las partículas de cobre y su capacidad de liberación, determinando que ambos compuestos, acetato monohidratado y nitrato trihidratado de cobre, son capaces de eliminar bacterias resistentes.

En la última década ha existido un aumento tanto en la proporción y en número absoluto de patógenos bacterianos que presentan multi-resistencia a los agentes antimicrobianos, organizaciones tales como Centro de Enfermedades Infecciosas de Atlanta (CDC), el Centro Europeo para la prevención y control de enfermedades (ECDC) y la Organización Mundial de la Salud (OMS), están considerando las infecciones causada por bacterias resistentes a múltiples fármacos (MDR) como una enfermedad global emergente y un importante problema en salud pública.

De acuerdo a lo anterior, la necesidad de encontrar nuevos productos antibacterianos ha llevado a una serie de iniciativas, entre las que destaca la "promoción de la investigación y el desarrollo de nuevas estrategias antimicrobianas $y$ agentes antibacterianos" 38 .

Es materia de futuros estudios, evaluar si con estos dos compuestos estudiados en el presente trabajo se obtienen resultados similares in vivo; lo que permitiría proponer a este grupo de investigación, una aplicación diferente a la utilización del cobre en áreas hospitalarias, atención primaria y ambulatorias ${ }^{39-40}$.

Agradecimientos: Los autores agradecen a la Comisión Nacional de Investigación Científica y Tecnológica (CONICYT), Ministerio de Educación Chile, por financiar el proyecto FONDEF- 
IDEA número de folio: CA13i10225, cuyos datos parciales son presentados en este documento.

\section{Referencias}

1. Mustoe T. Undertanding chronic wounds: a unifying hypothesis on the pathogenesis and implications for therapy. Am J Surg 2004; 187 (5A): 65-70.

2. Beebe-Dimmer J, Pfeifer J, Engle J, Schottenfeld D. The Epidemiology of Chronic Venous Insufficiency andVaricose Veins. Ann Epidemiol 2005; 15 (3): 175-84.

3. Aburto I, Morgado P. Gestión en manejo avanzado de heridas y úlceras en Chile. Rev Chil heridas \& Ostomias 2010; 1: 5-12.

4. Aburto I, Morgado P. guía clínica curación avanzada de las úlceras de pie diabético. Ministerio de Salud. Gobierno de Chile. Programa de salud cardiovascular. Santiago. 2005 (consultado en enero de 2012). http:// www.redsalud.gov.cl/archivos/saludcardiovascular/ Guia_clinica_Curacion_Pie_Diabetico.pdf

5. Gardner SE, Frantz RA, Doebbeling BN. The validity of the clinical signs and symptoms used to identifylocalized chronic wound infection. Wound Repair Regen 2001; 9 (3): 78-186.

6. Oyibo SO, Jude EB, Tarawnweh Y, Nguyen HC, Armstrong DG, Harkless LB. The effects of ulcer size and site, patient's age, sex and type and duration of diabetes on the outcome of diabetic foot ulcers. Diabet Med 2001; 18: 133-8.

7. Gottrup F. Management of the diabetic foot: surgical and organizational aspects. Horm Metab Res 2005; 37 Suppl 1: 69-75.

8. Iribarren O, Passi G, Aybar N, Ríos P, González L, Rojas $\mathrm{M}$, et al. Pie diabético: Evolución en una serie de 121 pacientes. Rev Chil Cir 2007; 59 (5): 37-341.

9. Bergin SM, Wraight P. Apósitos para heridas y agentes tópicos con plata para el tratamiento de la úlcera del pie diabético (Revisión Cochrane traducida). En: La Biblioteca Cochrane Plus, 2007, Número 4. Oxford: Update Software Ltd. Disponible en: http://www.updatesoftware.com

10. Van Hesselt P, Gashe BA, Ahmad J. Colloidal silver as an antimicrobial agent: fact or fiction? J Wound Care 2004; 13 (4): 154-5.

11. Ministerio de Salud. Gobierno de Chile. Encuesta Nacional de salud ENS 2009-2010, (consultado el 24 demarzo 2012), disponible en http://www.minsal.gob.cl/portal/ url/item/bcb03d7bc28b64dfe040010165012d23.pdf

12. Calderón A. Perfil de pacientes en curación avanzada de úlceras de pie diabético, en el consultorio Pudahuel poniente de la Región Metropolitana de Santiago. Rev Chil Heridas \& Ostomias 2011; 2: 78-82.

13. Thomas S, McCubbin P. An in-vitro analysis of the antimicrobial properties of 10 silver-containing dressings. J Wound Care 2003; 12 (8): 305-8.

14. Aburto I, Juliet C, Salas C. El efecto de la polimihexanida con betaína en el biolfilm enlas úlceras venosas. 2010 . Consultado el 15 marzo de 2012 disponible en (http:// www.inheridas.cl/wpcontent/uploads/Revision-Resumen-Prontosan.pdf ).

15. Perelshtein I, Applerot G, Perkas N, Wenhrschuetz-Sigl E, Hasmann A, Guebitz G, et al. CuO-cotton nanocomposite: Formation, morphology, and antibacterial activity. Surface \& Coatings Technology 2009; 204: 54-7.

16. Ameer A, Arham A, Mohammad O, Mohammad SK, Sammi H, Adnan M. Antimicrobial activity of metal oxide nanoparticles against Gram-positive and Gram-negative bacteria: a comparative study. Int J Nanomedicine 2012; 7: 6003-9.

17. Faúndez G, Troncoso M, Navarrete P, Figueroa G. Antimicrobial activity of copper surfaces against suspensions of Salmonella enterica and Campylobacter jejuni. BMC Microbiol 2004; 4: 19.

18. Itoh S, Kim HW, Nakagawa O, Ozumi K, Lessner SM, Aoki $\mathrm{H}$, et al. Novel role of antioxidant-1 (Atox1) as a copper-dependent transcription factor involved in cell proliferation. J Biol Chem 2008; 283 (14): 9157-67.

19. Borkow G, Gabbay J, Dardik R, Eidelman AL, Lavie Y, Grunfeld Y, et al. Molecular machaninms of enhanced wound healing by cooper oxide-impregnated dressings. Wound Rep Reg 2010; 18: 266-25.

20. Cioffi N, Torsi L, Ditarantano G, Tantalillo L, Ghibelli L, Sabbatini L, et al. Copper nanoparticle/polymer composites with antifungal and bacteriostatic properties. Chem Mater 2005; 17 (21): 5255-62.

21. Zhang W, Zhang YH, Ji JH, Zhao J, Yan Q, Chu PK. Antimicrobial properties of copper plasma-modified polyethylene. Polymer 2006; 47: 7441-5.

22. Palza H, Gutiérrez S, Delgado K, Salazar O, Fuenzalida V, Ávila J, et al. Toward tailor-made biocide materials based on polypropylene /copper nanoparticles. Macromolecular rapid communications 2009; 31 (6): 563-7.

23. Delgado K, Quijada R, Palma R, Palza H. Polypropylene whit embedded copper metal or copper oxide nanoparticles as a novel plastic antimicrobial agent. Lett Appl Microbiol 2011; 53 (1): 50-4.

24. Zhang E, Li F, Wang H, Liu J, Wang C, Li M, Yang K. A new antibacterial titanium-copper sintered alloy: Preparation and antibacterial property. Mater Sci Eng C Mater Biol Appl 2013; 33 (7): 4280-7.

25. Achard ME, Stafford SL, Bojil NJ, Chartres J, Bernhardt 
PV, Schembri MA, et al. Copper redistribution in murine macrophages in response to Salmonella inection. Biochem J 2012; 444: 51-7.

26. Gosau M, Bürgers R, Vollkommer T, Holzmann T, Prantl L. Effectiveness of antibacterial copper additives in silicone implants. J Biomater Appl 2013; 28 (2): 18798.

27. Amachawadi RG, Scott HM, Alvarado CA, Mainini TR, Vinasco J, Drouillard JS, et al. Occurrence of the transferable copper resistance gene tcrB among fecal enterococci of US feedlot cattle fed coppersupplemented diets. Appl Environm Microbiol 2013; 79: 4369-75.

28. Rowland J, Niederweis M. A multicopper oxidase is required for copper resistance in Mycobacterium tuberculosis. J Bacteriol 2013; 195: 3724-33.

29. Ministerio de Salud, Chile. Departamento de Epidemiología \& Departamento de Promoción de la Salud. [internet]. 2000 [citado el 27 de mayo de 2016]; disponible en: http://www.ine.cl/canales/chile_estadistico/ calidad_de_vida_y_salud/calidadvida/finalnacional.pdf

30. Clinical Laboratory Standards Institute (CLSI). M02-A10. Performance standards for antimicrobial disk susceptibility test; aproved standard. Tenth edition. Pensylvania, 2009.

31. Franks P, Barker J, Collier M, et al. Management of Patients with Venous Leg Ulcer: Challenges and Current Best Practice. J Wound Care 2016; 25 Suppl 6: S1-S67.

32. Europan Wound Management Association (EWMA). Position Document: identifying criteria for wound infection. London: MEP Ltd, 2005.

33. O’Meara S, Al-Kurdi D, Ologun Y, Ovington LG, Martyn-St James M, Richardson R. Antibiotics and antiseptics for venous leg ulcers (Review) Revision Cochra- ne 2014 [citado el 27 de marzo de 2016]; disponible en: http://www.cochrane.org/CD003557/WOUNDS_antibiotics-and-antiseptics-to-help-healing-venous-leg-ulcers.

34. Stickler DJ. Susceptibility of antibioticresistant Gram-negative bacteria to biocides: a perspective fron the study of catheter biofimls. J Appl Micrbiol 2002; 92 s: $163 \mathrm{~s}-70 \mathrm{~s}$.

35. Gottrup F, Apelqvist J, Bjarnsholt T, Cooper R, Moore Z, Peters EJ, et al. EWMA Document: Antimicrobials and Non-healing Wounds-Evidence, Controversies and Suggestions. J Wound Care 2013; 22 (5 Suppl.): S1- S92.

36. Stickler DJ. Susceptibility of antibiotic resistant Gram-negative bacteria to biocides: a perspective from the study of catheter biofimls. J Appl Micrbiol 2002; 92 s: $163 \mathrm{~s}-70 \mathrm{~s}$.

37. Grass G, Rensing C, Solioz M. Metallic copper as an antimicrobial surface. Appl Environ Microbiol 2011; 77 (5): 1541-7.

38. Roca I, Akova M, Baquero F, Carlet J, Cavaleri M, Coenen S, et al. The global threat of antimicrobial resistance: science for intervention. New Microbes New Infect 2015; 6: 22-9.

39. Prado JV, Vidal AR, Durán TC. [Application of copper bactericidal properties in medical practice]. Rev Med Chile 2012; 140: 1325-1332.

40. Rivero P, Brenner P, Nercelles P. Impacto del cobre en la reducción de infecciones intrahospitalarias, mortalidad y gasto en antimicrobianos en una unidad de cuidados intensivo de adultos. Rev Chil Infectol [internet]. 2014 jun [citado el 27 de mayo de 2016]; 31 (3): 274-9. disponible en: http://www.scielo.cl/scielo.php?script=sci_arttext\&pid=s0716-10182014000300004\&lng=es 